\title{
The Factors that Contribute to Change in Evaluation after Co- Occurrence with Evaluative Categorisation
}

\section{Yoav Bar-Anan ${ }^{\text {a* }}$}

After a neutral stimulus co-occurs with an affective stimulus, the evaluation of the neutral stimulus shifts toward the valence of the affective stimulus. The present research tested what factors contribute to evaluation change when, while observing the co-occurrence, people also categorise the affective stimuli. In Experiment 1, categorisation by valence caused stronger change in the evaluation of the neutral stimulus than non-evaluative categorisation, but only when categorisation for each valence was consistently mapped to the same motor response. In Experiment 2, co-occurrence with extreme affective stimuli during the categorisation task caused stronger change in the evaluation of the neutral stimulus than co-occurrence with mild affective stimuli. These results suggest that when neutral stimuli co-occur with evaluative stimuli and with an evaluative categorisation response, both types of co-occurrence contribute to evaluative change. In Experiment 3, induced contingency awareness did not increase evaluative change. This might suggest that contingency awareness does not contribute to the effect.

Keywords: Attitudes; Evaluation; Evaluative Conditioning; Categorisation; Attitude Formation

${ }^{a}$ Department of Psychology, Ben-Gurion University of the Negeve in Beer-Sheva, Israel;

*Correspondence should be addressed to: Yoav Bar-Anan, Department of Psychology, Ben-Gurion University in the Negev Be’er Sheva, Israel. E-mail: baranany@bgu.ac.il

Author's note: This project was supported by grants from the Israeli Science Foundation [779/16], and from Project Implicit Inc. and the United States - Israel Binational Science Foundation [2013214]. 
RUNNING HEAD: CONTINGENCY IN EVALUATIVE CATEGORISATION

\section{The factors that contribute to change in evaluation after co-occurrence with evaluative categorisation}

People constantly make choices about stimuli in the environment: they choose whether to focus on an object or ignore it, pursue it or escape, keep it or throw it away. Evaluation informs many of these choices. Usually, people approach stimuli they evaluate as positive and avoid stimuli they evaluate as negative. For that reason, evaluation is key for making choices, and studying what factors influence evaluation is informative for understanding human behaviour. One factor that influences evaluation is stimulus co-occurrence. People prefer stimuli that co-occurred with positive stimuli over stimuli that co-occurred with negative stimuli. This is the Evaluative Conditioning (EC) effect (De Houwer, Thomas, \& Baeyens, 2001; Walther, Weil, \& Dusing, 2011).

EC has been usually demonstrated in tasks that require passive perception of stimulus co-occurrence, or with distracting tasks in which the co-occurring stimuli-the neutral and the affective stimuli-were irrelevant to the task's goal (Olson \& Fazio, 2001). Therefore, there is relatively little knowledge about the effect of stimulus cooccurrence in more complex contexts that involve other experiences that might influence evaluation. It is possible that $\mathrm{EC}$ is not an important factor for evaluation when other evaluatively-relevant factors are available. Alternatively, the assimilative effect after stimulus co-occurrence might be stronger in more complex contexts, especially in cases that require people to act on the affective stimuli, rather than only observe them. Motivated by these questions, the present research examined a few factors that might 
modulate changes in evaluation when neutral stimuli co-occur with affective stimuli, while the affective stimuli are categorised evaluatively.

Because people tend to approach positive stimuli and avoid negative stimuli, categorisation of stimuli as favourable or unfavourable (i.e., evaluative categorisation) is ubiquitous. A neutral stimulus (conditioned stimulus - CS) that co-occurs with an affective stimulus (unconditioned stimulus - US) probably also often co-occurs with an evaluative categorisation of the affective stimulus. For instance, neutral stimuli in a restaurant that serves delicious food (e.g., the restaurant's décor and personnel) co-occur with positive stimuli (the delicious food) and with categorisation of the food as positive (prompted by a server's question). In such contexts, co-occurrence with the categorisation response might facilitate the change in the evaluation of the CS. This seems likely based on previous research (Gast \& Rothermund, 2011a) that found that co-occurrence of neutral stimuli with an evaluative response leads to an assimilative effect on the evaluation of the neutral stimuli. In one study, participants who categorised CS-US pairs by valence showed EC (an assimilation of the CS evaluation toward the initial US valence), even after the US valence changed due to new information. In another study that did not involve any stimulus co-occurrence, participants evaluated CSs more positively if the CSs co-occurred with voicing the word Likeable (a positive evaluative response) than if the CSs co-occurred with voicing the word Unlikeable.

Another reason to assume that evaluative categorisation is a facilitating factor for an assimilative evaluative effect is that the categorisation focuses people on the evaluative dimension. Previous research (Gast \& Rothermund, 2011b) found that focus 
on evaluation facilitates EC. Participants who rated CS-US pairs by valence showed stronger EC than participants who rated the pairs by geographical location or by age. In another study, only filler stimuli, and not the CS and US were categorised. Categorisation of the filler stimuli by valence led to EC, whereas categorisation by style (the stimuli were garments) did not lead to EC. These findings suggest that focus on valence might contribute to a change in the evaluation of CS when the USs are evaluatively categorised.

\section{The Present Experiments}

To learn more about the factors that modulate change in evaluation in the context of US categorisation, the present study adapted a previous procedure (Schmidt \& De Houwer, 2012) that linked co-occurrence with categorisation. On each trial, pairs of a nonsensical word and an affective image appeared together on the screen. Participants' task was to categorise the image. The CS words co-occurred with images of a single valence. After the task, participants rated the words.

The present research pursued a few questions. In Experiment 1, I examined whether categorisation by valence leads to a stronger EC than categorisation on a less evaluative dimension (gender). That would conceptually replicate the findings that focus on valence (Gast \& Rothermund, 2011b) and that co-occurrence with evaluative response (Gast \& Rothermund, 2011a) contribute to evaluative change, although it would not separate between these two factors. For more information on the effect, in Experiment 1, the categorisation's mapping to key responses (left versus right) was either constant throughout the task, or randomly assigned on each trial. When the categorisation's motor response is the same throughout the task, the CS co-occurs with a motor response that 
comes to signify valence. Past research found that co-occurrence of a CS with a motor response that comes to signify a certain valence pushes the evaluation of the CS toward the valence signified by the motor response (Laham, Kashima, Dix, Wheeler, \& Levis, 2014). I examined whether that kind of pairing has any role in changes in the evaluation of the CS, when people categorise the US.

To anticipate the results, in Experiment 1 the change in the CS evaluation was stronger when the categorisation was evaluative than non-evaluative, but only if the CS co-occurred with the same motor response throughout the task. When the evaluative meaning of the motor responses changed throughout the categorisation task, the change in the CS evaluation was not stronger than when participants categorised USs by evaluation than when US categorisation was by gender. These results suggest that pairing with a response, rather than focus on valence, contributed to the effect.

Based on Experiment 1's results, the next question, investigated in Experiment 2, was whether stimulus co-occurrence has any contribution to the evaluation change when the CSs co-occur with a motor response that comes to signify one valence throughout the task. As discussed earlier, it is possible that EC does not occur when other evaluativerelevant experiences are available. To examine that possibility, in Experiment 2, some CSs co-occurred with extreme USs, whereas other CSs co-occurred with relatively mild USs. If stimulus co-occurrence still contributes to the change in the CS evaluation, the change should be stronger for CSs that co-occurred with extreme valence than for CSs that co-occurred with mild valence. 
Finally, Experiment 3 examined another factor that might contribute to evaluative change in a categorisation task: contingency awareness. There is evidence that knowledge that a CS would co-occur with USs of a certain valence changes the evaluation of the CS, even without experiencing the actual co-occurrence (De Houwer, 2006). There is no conclusive evidence that experiencing stimuli co-occurrence can lead to EC without awareness of the co-occurrence (Sweldens, Corneille, \& Yzerbyt, 2014, for a review). Therefore, awareness of the co-occurrence is considered a possible factor that contributes to EC (De Houwer, 2009; Kattner, 2012). The evaluative categorisation task seems apt for making participants aware of co-occurrence with valence. The participants are motivated by the task instructions and error feedback to quickly determine whether the US is positive or negative. Awareness of the CS-US contingency might facilitate those goals. Therefore, awareness of the contingency might be a strong mediating factor in the assimilative effect investigated in the present work.

One weakness in most previous research on the role of awareness in EC is that awareness was investigated by measuring memory of contingency at the end of the experiment. However, contingency memory could be the result of change in evaluation rather than the cause for the change (Bar-Anan \& Amzaleg-David, 2014; Gawronski \& Walther, 2012). Moreover, EC without memory could reflect forgetting of the contingency rather than lack of awareness during acquisition. To avoid that problem, Experiment 3 manipulated contingency awareness by providing full information, partial information or no-information about the contingency, in advance. If contingency awareness is a contributing factor to the assimilative effect, providing the participants better knowledge of the contingency should increase the assimilative effect. If the results 
would show that induced contingency awareness increases the assimilative effect, it would provide evidence that knowledge can be a contributing factor in the present task ${ }^{1}$.

\section{Experiment 1}

\section{Method}

Participants. Participants in all experiments were volunteers who completed the study over the Internet at the Project Implicit website (Nosek, 2005). 683 participants $\left(426\right.$ Females, $\left.\mathrm{M}_{\mathrm{age}}=37.5, \mathrm{SD}_{\mathrm{age}}=13.7\right)$ started the study, and 454 participants completed all the measures $^{2}$. The analyses did not include 11 participants with error-rates above $27 \%-2$ SDs above the average error-rate in the categorisation task. The final sample had 443 participants $\left(273\right.$ Females, $\left.M_{\text {age }}=37.7, S D_{\text {age }}=13.9\right)$. The sample provided $95.7 \%$ power to detect an interaction of effect of $\eta_{p}{ }^{2}=.03$, and $80 \%$ for $\eta_{p}{ }^{2}=$ .018 .

Materials. The eight USs were images of four men and four women - four taken from the International Affective Picture System (IAPS) CD-ROM (Lang, Bradley, \& Cuthbert, 1999), and four found on the Internet (see at osf.io/gbvzr/). There were two positive and two negative images for each sex. The CSs were five nonsense words

\footnotetext{
${ }^{1}$ I report all data exclusions, manipulations, and measures. For each experiment, I determined sample size before data collection, with the goal to attain at least $80 \%$ power for a small effectsize. Decisions to stop collecting data did not depend on the obtained results. Materials, data and analysis scripts are in osf.io/7n99c/.

${ }^{2}$ The web platform allowed for larger samples and wider heterogeneity (e.g., in participants' age) in comparison to most lab studies. However, volunteers outside the lab drop out of experiments more often than lab participants. Because the program recorded the between-participants conditions in Experiments 1 and 3 only after they finished the acquisition task, there is no information on the effect of those conditions on drop-out rate.
} 
printed in capital letters: BAUBED, EBELSA, QUESTL, HANE, and THEET. For each participant, the words were randomly assigned to two positive CSs $\left(\mathrm{CS}_{\mathrm{pos}}\right)$, two negative CSs $\left(\mathrm{CS}_{\text {neg }}\right)$, and one filler CS.

Procedure. All the experiments were web studies programmed with Minno.js (Zlotnick, Dzikiewicz, \& Bar-Anan, 2015). The consent form indicated that studies in Project Implicit are about attitudes, preferences and memory. Participants read that they would complete a sorting task and answer a few question. Participants first completed the categorisation task, with a 2 (categorisation: by gender, by valence) X 2 (response-key assignment: constant throughout the task, randomly assigned in each trial) design. Afterwards the participants rated the CSs.

Pairing. The pairing occurred in a categorisation task. Participants either categorised the USs as Male/Female or Pleasant/Unpleasant. The response keys were either randomly assigned to one category in the beginning of the task and kept constant throughout the task, or randomly assigned to categories in each trial. The instructions before the task were:

Put your middle or index fingers on the E and I keys of your keyboard. Words and images will appear on the screen. Ignore the words and categorise the images as Pleasant [Female] or Unpleasant [Male].

At the top of the screen, you will see which category requires a left response and which category requires a right response.

Press E for left response, and I for a right response.

[The categories sometimes change locations, so always look at the top of the screen to know which category requires the left key and which category requires the right key.]

If you make a mistake, a red X will appear. You will have about one second to categorise the image. GO AS FAST AS YOU CAN while making as few mistakes as possible. 
The trial sequence started with a 500ms display of the category labels (to communicate key assignment), followed by $250 \mathrm{~ms}$ display of the prime word, appearing twice, at the top and bottom of the experiment window. Next, the target image appeared instead of one of the two displays of the prime word. If the participant did not press one of the two response keys 3300ms after the appearance of the target stimulus, the message '!!!PLEASE RESPOND FASTER!!!' appeared for 500ms and the trial ended. A red X appeared for 250ms after incorrect responses. Between trials, a blank screen appeared for duration selected randomly for each trial, ranging from $300 \mathrm{~ms}$ to $900 \mathrm{~ms}$. In each of the four blocks, each CS occurred twice with each of the four USs of one valence (same valence throughout the task), whereas the filler stimulus occurred once with each of the eight USs. In total, each block had 40 trials, and each CS co-occurred 32 times with USs of one valence.

Rating. Rating had four rounds. Each round presented the same question for each of the five CSs, presented in a random order. The question in the first round was During the sorting task, how pleasant did you feel that this word was? The question in the second round was Based only on what you feel right now, how much do you like this word? The last two rounds were presented in a random order, one with the question If this word had a meaning, how positive do you feel that the meaning would have been? And one with the question Based only on visual appearance, how pleasant is this word's appearance? The response was on a 9-point scale ranging from 1: Not at all to 9: Extremely.

\section{Results}


In the analyses of the present experiments, when inference from no effect was informative or when the $p$ value was only slightly below .05, I searched more information for statistical inference by computing Bayes factors (using JASP, 2017, version 0.8.01), with the default Jeffreys prior (Rouder, Morey, Speckman, \& Province, 2012). Strength of the evidence was interpreted based on suggestions by Jeffreys (Appendix B, 1961).

Table 1. Experiment 1: The effect of the manipulation on task performance

\begin{tabular}{|c|c|c|}
\hline Error-rate & Categories Switched Sides & Constant Categories Sides \\
\hline Gender & $0.057(0.035)$ & $0.039(0.029)$ \\
\hline Evaluation & $0.074(0.055)$ & $0.046(0.038)$ \\
\hline \multicolumn{3}{|c|}{ Categorisation task: $F(1,439)=11.90, p=.001, \eta_{\mathrm{p}}^{2}=.03$} \\
\hline \multicolumn{3}{|c|}{ Key-assignment: $F(1,439)=33.90, p<.001, \eta_{\mathrm{p}}^{2}=.07$} \\
\hline \multicolumn{3}{|c|}{ Interaction: $F(1,398)=2.72, p=.100, \eta_{\mathrm{p}}^{2}=.01$} \\
\hline Latency & Category sides Switched & Category sides Constant \\
\hline Gender & $942(189)$ & $650(118)$ \\
\hline Evaluation & $1020(211)$ & $683(115)$ \\
\hline \multicolumn{3}{|c|}{ Categorisation task: $F(1,439)=12.50, p=.001, \eta_{\mathrm{p}}^{2}=.03$} \\
\hline \multicolumn{3}{|c|}{ Key-assignment: $F(1,439)=404.11, p<.001, \eta_{\mathrm{p}}^{2}=.48$} \\
\hline Interaction: & $.05, p=.153, \eta_{\mathrm{p}}^{2}<.01$ & \\
\hline
\end{tabular}

Task performance. Table 1 displays average error-rate and latency in each of the four conditions, and the results of a 2 (categorisation: valence, gender) X 2 (response-key assignment: constant, random) ANOVA on those variables. Participants were faster and more accurate when key assignment was constant throughout the task, and when they categorised by gender rather than by valence. 
Evaluation. The average evaluations of each two CSs that had the same role (e.g., the two $\mathrm{CS}_{\text {pos }} \mathrm{S}$ ) were submitted to a 2 (categorisation) x 2 (response-key assignment) x 2 (Paired-valence) x 4 (question) repeated measures ANOVA. Table 2 displays the relevant means. Figure 1 illustrates the effect of paired-valence (the difference between rating of $\mathrm{CS}_{\text {pos }}$ and $\mathrm{CS}_{\text {neg }}$ ) as a function of the three other factors.

Table 2. Experiment 1: Average rating of CSs by categorisation-task, key-assignment condition, and US valence.

\begin{tabular}{lcccccccc}
\hline Task & \multicolumn{3}{c}{ Evaluation } & \multicolumn{3}{c}{ Gender } \\
Key-Assignment & \multicolumn{2}{c}{ Constant } & \multicolumn{2}{c}{ Switching } & Constant & \multicolumn{2}{c}{ Switching } \\
US & Neg & Pos & Neg & Pos & Neg & Pos & Neg & Pos \\
\hline Question & & & & & & & & \\
Pleasant & 3.480 & 4.847 & 3.803 & 4.542 & 3.912 & 4.338 & 3.695 & 4.184 \\
& $(1.723)$ & $(2.066)$ & $(1.568)$ & $(1.723)$ & $(1.727)$ & $(1.873)$ & $(1.787)$ & $(1.775)$ \\
Like & 3.644 & 4.911 & 4.038 & 4.399 & 3.934 & 4.540 & 3.718 & 4.259 \\
& $(1.771)$ & $(1.992)$ & $(1.583)$ & $(1.62)$ & $(1.706)$ & $(1.747)$ & $(1.728)$ & $(1.728)$ \\
Looks & 3.832 & 4.901 & 4.408 & 4.613 & 4.390 & 4.798 & 4.203 & 4.557 \\
& $(1.696)$ & $(1.912)$ & $(1.454)$ & $(1.479)$ & $(1.555)$ & $(1.709)$ & $(1.823)$ & $(1.716)$ \\
Meaning & 3.767 & 5.153 & 4.235 & 4.597 & 4.419 & 4.787 & 4.144 & 4.420 \\
& $(1.612)$ & $(1.936)$ & $(1.465)$ & $(1.47)$ & $(1.602)$ & $(1.575)$ & $(1.866)$ & $(1.725)$
\end{tabular}




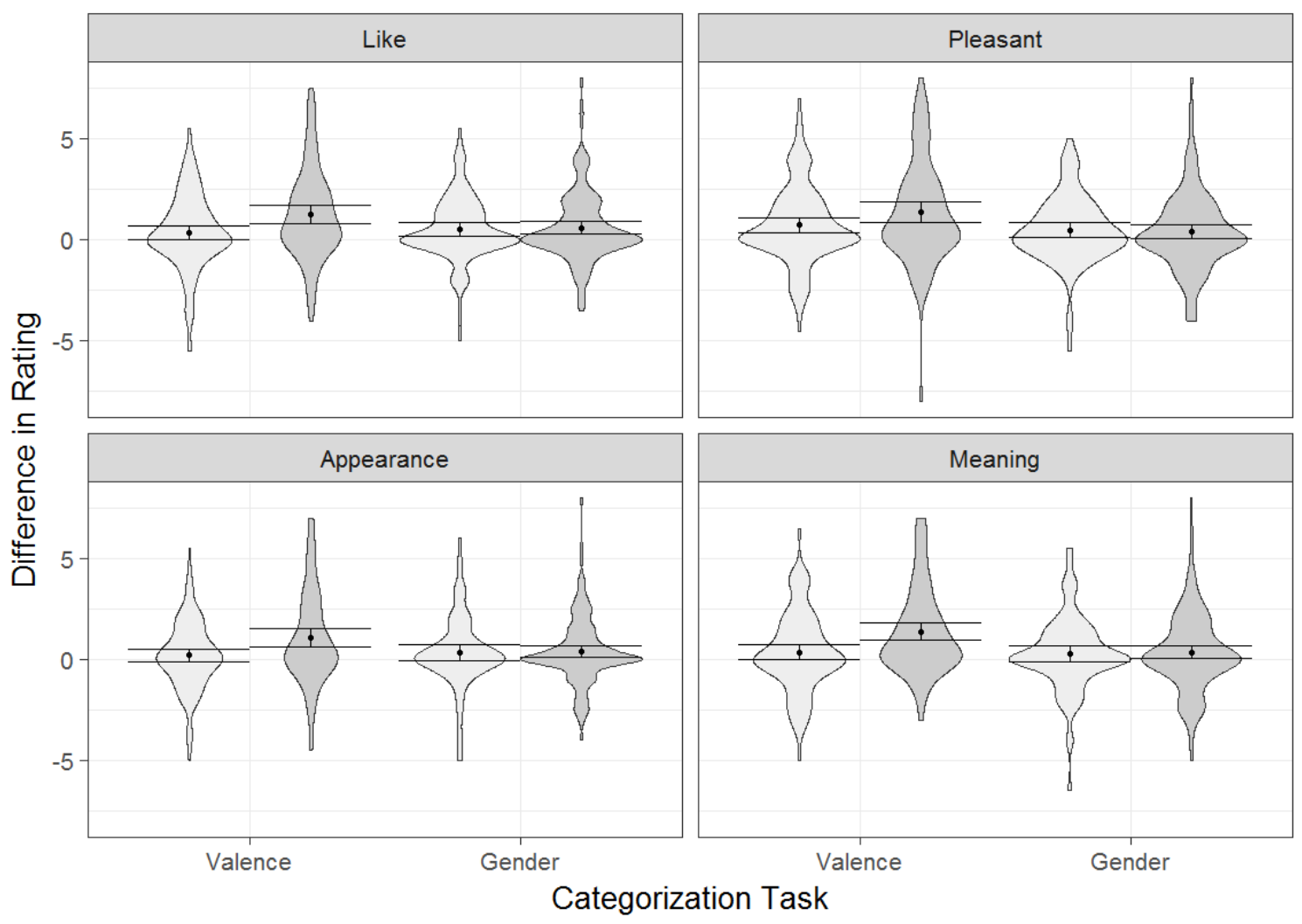

Key Assignment Switching
Constant

Figure 1. The effect of paired valence (difference between $C S_{\text {pos }}$ and $C S_{\text {neg }}$ rating) by question (quadrants), categorisation task (x-axes), and key-assignment (colour); Black dot $=$ mean, Error bars $=95 \%$ confidence interval, violins $=$ vertical density plots .

In each categorisation condition, in each switching condition and each question, participants rated the $\mathrm{CS}_{\text {pos }}$ more positively than the $\mathrm{CS}_{\mathrm{neg}}$, leading to a main effect of paired-valence, $F(1,439)=63.27, p<.001, \eta_{p}{ }^{2}=.13$. That effect was moderated by keyassignment condition, $F(1,439)=7.87, p=.005, \eta_{p}{ }^{2}=.02$, reflecting stronger effect of US valence when the position of the categories was constant, $F(1,235)=54.42, p<.001$, $\eta_{p}{ }^{2}=.19$, than when position switched randomly, $F(1,204)=14.86, p<.001, \eta_{p}{ }^{2}=.07$. The US valence effect was also moderated by categorisation task, with a stronger effect when the categorisation was evaluative, $F(1,218)=47.49, p<.001, \eta_{p}{ }^{2}=.18$, than when the categorisation was by gender, $F(1,221)=17.41, p<.001, \eta_{p}{ }^{2}=.07$. That moderation 
might reflect the effect of focus on valence or of co-occurrence with an evaluative categorisation response.

There was also a small three-way interaction between US-valence, categorisation task, and key-assignment, $F(1,439)=6.40, p=.012, \eta_{p}{ }^{2}=.01, B F_{10}=3.79($ the Bayes factor is interpreted as moderate evidence in favour of the moderation). As Figure 1 illustrates, there was an equal effect of paired-valence when participants categorised by gender and the response positions switched randomly $\left[F(1,86)=7.23, p=.009, \eta_{p}{ }^{2}=\right.$ $.08]$, when participants categorised by gender and the response positions did not switch randomly $\left[F(1,135)=11.52, p=.001, \eta_{p}{ }^{2}=.08\right]$, and when participants categorised by valence and the response positions switched randomly $\left[F(1,118)=8.21, p=.005, \eta_{p}{ }^{2}=\right.$ .07] - all clearly smaller than the paired-valence effect when participants categorised by valence and the response positions did not switch throughout the task $[F(1,100)=39.09$, $\left.p<.001, \eta_{p}{ }^{2}=.28\right], F(1,441)=20.15, p<.001, \eta_{p}{ }^{2}=.04$, for the contrast. In other words, the three-way interaction reflected a stronger moderation by switching condition when participants categorised by valence, $F(1,218)=12.17, p=.001, \eta_{p}{ }^{2}=.05, B F_{10}=$ 43.08, than when participants categorised by gender, $F(1,221)=0.05, p=.831, \eta_{p}^{2}<$ $.01, B F_{01}=10.00$. The $\mathrm{BF}_{01}$ value reflected strong evidence that key assignment did not moderate the paired-valence effect when participants categorised by gender. This lack of moderation is important because it suggests that switching keys throughout the task did not put a strain on participants' cognitive resources that decreased the effect of paired valence on evaluation. Why, then, did key assignment consistency moderate the effect of paired-valence when participants categorised the USs by valence? Perhaps evaluation change was larger when the key assignment was constant than when it varied throughout 
the evaluative categorisation task because a constant key assignment paired each motor response with one certain valence. It seems that pairing the CS with a motor response mapped to valence is a key factor that contributes to change in the CS evaluation.

Comparing the two conditions in which the key-assignment was randomly assigned in each trial, evaluative categorisation did not produce a stronger paired-valence effect than gender categorisation, $F(1,204)=0.00, p=.967, \eta_{p}{ }^{2}<.01, B F_{01}=6.28$. The Bayes factor suggested moderate evidence that the categorisation dimension did not moderate the result. In other words, focus on valence did not increase the effect of paired valence, nor did the co-occurrence with an evaluative categorisation response that was not mapped consistently to a motor response ${ }^{3}$.

In summary, Experiment 1 found evidence that pairing stimuli with evaluative categorisation that requires a constant motor response increases the effect of cooccurrence with valence on evaluation. Focus on the evaluative dimension did not increase the effect of co-occurrence on evaluation. The results also suggest that stimulus co-occurrence in the context of a categorisation task can still have an effect on evaluation, even when the categorisation is not directly evaluative. Nevertheless, it is still possible that when the stimuli co-occurred with a motor response that was paired with evaluative categorisation the pairing with the response was the sole reason for the assimilative effect on evaluation. Perhaps pairing with a response replaces the effect of

\footnotetext{
${ }^{3}$ In the main ANOVA, there were also a main effect for question type, $F(3,1317)=21.04, p<.001, \eta_{p}{ }^{2}=$ .05 , and question type moderated the paired-valence effect, $F(3,1317)=3.66, p=.014, \eta_{p}{ }^{2}=.01$. However, these effects seem less relevant. As Figure 1 illustrates, the pattern of results was slightly different for the question about how pleasant participants felt during the task toward each word. However, the other questions seem more relevant for effects on evaluation, and they all showed a similar pattern (the one described in the previous paragraph).
} 
stimulus co-occurrence rather than adds to it. In that case, stimulus co-occurrence influenced evaluation in three of the four between-participants conditions in Experiment 1, but not in the condition that paired the stimuli with a motor response (hit the left or the right response key) that was consistently paired with one valence. That is an important possibility because it would suggest that EC is limited and does not occur when there are other factors that affect evaluation. Experiment 2 examined whether stimulus cooccurrence has any effect when the CS is paired with a constant motor response that indicates evaluative categorisation.

\section{Experiment 2}

Experiment 2 examined whether extremity of the valence of the US moderates the effect of pairing in the context of a categorisation task. Each CS co-occurred with a single US - very positive, mildly positive, mildly negative, or very negative. The categorisation task was similar to the evaluative categorisation in Experiment 1 (with no random switching of the key responses). A moderation by US valence would suggest that stimulus co-occurrence still affects evaluation, even when the CS is also paired with a constant motor response that comes to signify an evaluative categorisation of a certain valence.

\section{Method}

Participants. 957 participants (612 Females, $\left.\mathrm{M}_{\mathrm{age}}=34.5, \mathrm{SD}_{\mathrm{age}}=14.0\right)$ started the study, and 669 participants completed all the measures. Of those, the analyses did not include 17 participants with correct-response rates below $64 \%-2$ SDs below the average 
rate in the categorisation task. The final sample had 652 participants (418 Females, $\mathrm{M}_{\text {age }}$ $\left.=35.1, \mathrm{SD}_{\mathrm{age}}=13.8\right)$. The sample provided more than $99 \%$ power to detect a $\eta_{p}{ }^{2}=.03$ moderation effect, and $80 \%$ power for $\eta_{p}^{2}=.012$

Materials. The four extreme-valence USs were two positive and two negative images from the IAPS. The four mild-valence USs were black and white sketches found using Google Images search (available at osf.io/744y8/). The CSs were eight nonsense words printed in capital letters, chosen randomly for each participant from a list of ten words (BAUBED, EBELSA, QUESTL, HANE, THEET, PEEMING, DRIBS, HUBE, ODWOUN, OTAIR) and randomly assigned to the four CS conditions (two $\mathrm{CS}_{\text {pos-extreme, }}$ two $\mathrm{CS}_{\text {pos-mild, }}$ two $\left.\mathrm{CS}_{\text {neg-extreme, two }} \mathrm{CS}_{\text {neg-mild }}\right)$.

Procedure and Design. The consent form was the same as in Experiment 1. The categorisation task (acquisition) was identical to the one used in Experiment 1 in the valence condition that had constant key-responses assignment throughout the task, with the following modifications. Each of the eight CSs was randomly assigned to always cooccur with one of the eight USs. In each block, each CS co-occurred four times with the US, for a total of 32 trials in each block, and 16 pairings for each CS throughout the task. In the trial sequence, the prime appeared for $500 \mathrm{~ms}$, followed by the target appearing until response or until 2000ms passed. If participants responded incorrectly or did not respond in 2000ms, a red X message (for errors) or a Respond Faster message appeared for $600 \mathrm{~ms}$. The trial ended with a $250 \mathrm{~ms}$ black screen (the ITI).

Afterward, participants rated each of the 16 CS and US (random order - either all USs first, or all CSs first). The question was: How positive or negative are your feelings 
RUNNING HEAD: CONTINGENCY IN EVALUATIVE CATEGORISATION

toward [the word OTAIR/this image]? And the response scale was Extremely negative, Moderately negative, Slightly negative, Neutral, Slightly positive, Moderately positive, Extremely positive, coded 1 to 7.

The design was 2 (paired-valence: positive, negative; within participants) x 2 (US extremity: Extreme, Mild; within participants).

\section{Results}

Figure 2 displays the rating distributions of all 16 stimuli.

US evaluation. The extreme $\mathrm{US}_{\mathrm{pos}} \mathrm{S}$ were rated more positively $(M s=6.48,6.13$, $S D s=0.80,1.05)$ than the mild $\mathrm{US}_{\mathrm{pos}} \mathrm{s}(M s=5.65,5.65, S D s=0.97,1.00), t(651)=$ $10.059, p<.001, d=0.39$ (95\% CI: $0.28,0.50)$, for the comparison between the two closest stimuli. The extreme $\mathrm{US}_{\text {negs }}$ were rated more negatively $(M s=1.78,1.85, S D s=$ $1.11,1.07)$ than the mild $\mathrm{US}_{\mathrm{neg}}(M s=2.56,2.62, S D s=1.18,0.91), t(651)=15.637, p<$ $.001, d=0.61(95 \%$ CI: $0.50,0.72)$, for the comparison between the two closest stimuli.

CS evaluation. A 2 (paired-valence) x 2 (US extremity) repeated measures ANOVA found a main effect of paired-valence, $F(1,651)=83.14, p<.001, \eta_{\mathrm{p}}{ }^{2}=.11$, reflecting more positive evaluation of $\mathrm{CS}_{\mathrm{pos}}(M=4.09, S D=0.65)$ than $\mathrm{CS}_{\text {neg }}(M=3.75$, $S D=0.60)$. There was no main effect for extremity, $F(1,651)=0.04, p=.840, \eta_{\mathrm{p}}^{2}<.01$. A significant interaction, $F(1,651)=36.56, p<.001, \eta_{\mathrm{p}}{ }^{2}=.05$, reflected a stronger effect of paired-valence when the USs were extreme, $t(651)=9.75, p<.001, d=0.38(95 \% \mathrm{CI}$ : $0.27,0.49)$, than when the USs were mild, $t(651)=4.21, p<.001, d=0.16(95 \% \mathrm{CI}$ : $0.06,0.27)$. 

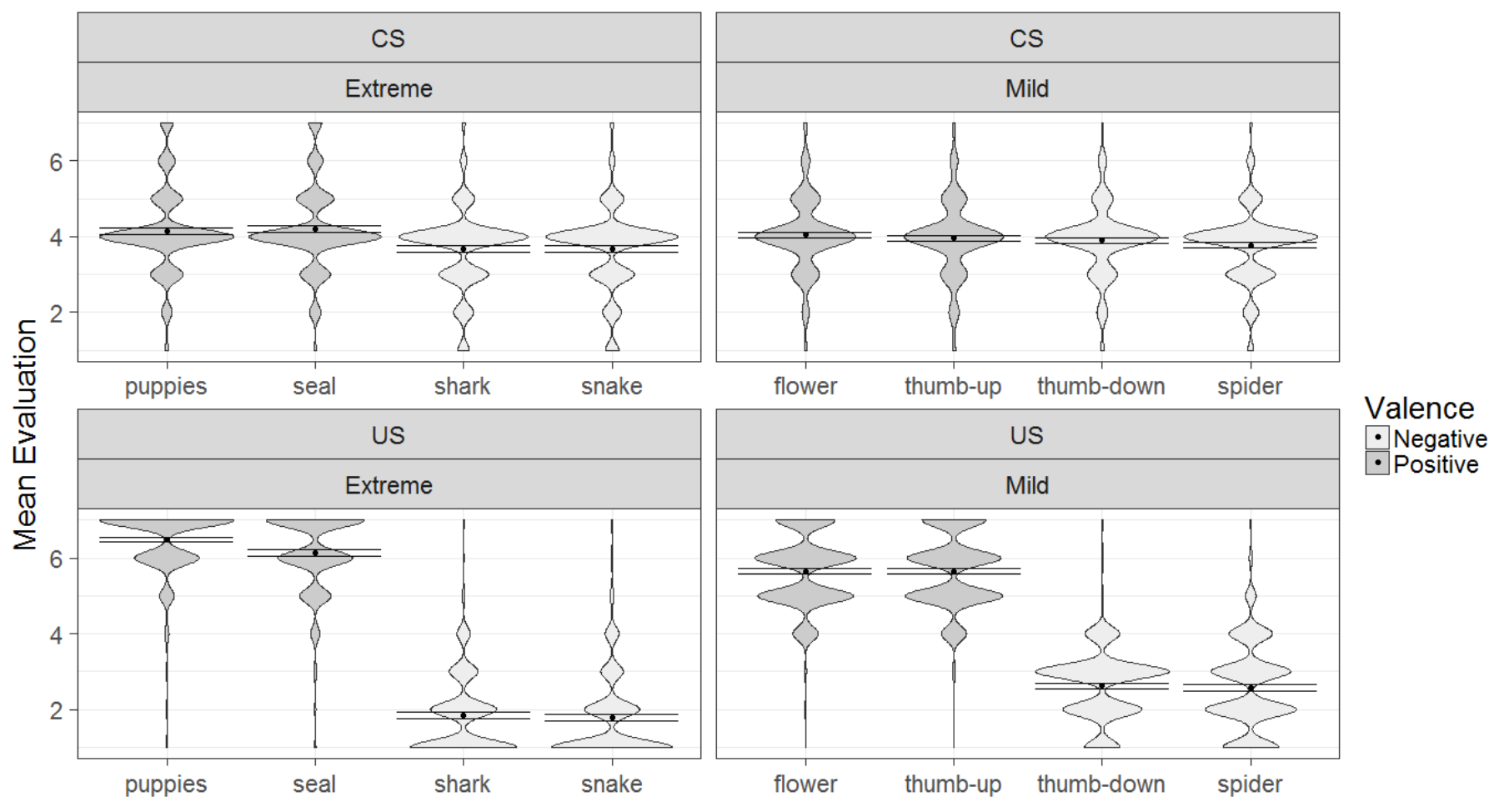

- Negative

- Positive

Figure 2. Rating of the CSs (top quadrants) and USs (bottom quadrants). The x-axis specifies the stimulus (for the USs) or the US with which the CS co-occurred in the task (for the CSs). Extreme stimuli are on the left quadrants and mild stimuli on the right quadrants; Black dot $=$ mean, Error bars $=95 \%$ confidence interval, violins $=$ vertical density plots.

One alternative account for why the co-occurring US valence had a stronger effect on evaluation when the USs were extreme rather than mild is that people made more correct responses when the USs were extreme than when the USs were mild. As a result, the CSs that co-occurred with more extreme USs also co-occurred with more evaluative responses that matched the US valence. In that case, rather than reflecting the effect of stimulus co-occurrence, the interaction reflected a different number of co-occurrence 
with the evaluative categorisation. In support of that possibility, the rate of correct responses was higher for extreme USs $(M=0.949, S D=0.060)$ than for mild USs $(M=$ $0.909, S D=0.079), t(651)=16.066, p<.0001, d=0.63$. The effect of US extremity on correct-response rate also slightly predicted the moderation of the paired-valence effect by US extremity, $r(652)=.113, p=.004^{4}$. This mild correlation, however, could be the result of many reasons - not only an effect of the number of correct responses and the paired-valence effect. For instance, individual differences in subjective affective reaction to the USs might influence both effects. To examine whether US extremity moderated the effect of paired-valence beyond the effect of US extremity on correct-response rate, I repeated the main ANOVA analysis with a subset of participants who did not make more correct responses after extreme US than after mild US (they made either the same number of correct responses after both US types, or more correct responses after mild US than after extreme US). These are participants who did not experience a larger number of pairings with the evaluative response for $\mathrm{CS}_{\text {extreme }}$ in comparison to $\mathrm{CS}_{\text {mild. }}$ The interaction effect in that ANOVA was still statistically reliable, $F(1,194)=4.26, p<$ $.001, \eta_{p}{ }^{2}=.02$, and reflected the same moderation pattern.

\section{Discussion}

Pairing with valence (US and response) had a stronger effect when the US valence was more extreme. This suggests that co-occurrence with affective stimuli has an assimilative effect on evaluation of a neutral stimulus even when the CS also predicts a

\footnotetext{
${ }^{4}$ The values for the effects were difference scores. One score was the difference between correct response rate to extreme USs and to mild USs. The other value for the correlation was computed from four average ratings: $\left(\mathrm{CS}_{\text {pos-extreme }}-\mathrm{CS}_{\text {neg-extreme }}\right)-\left(\mathrm{CS}_{\text {pos-mild }}-\mathrm{CS}_{\text {neg-mild }}\right)$.
} 
motor response that signifies an evaluative categorisation response. In concert, Experiments 1 and 2 suggest that when a stimulus predicts an affective stimulus and an evaluative categorisation response of the same valence, both pairings contribute to an assimilative effect on the CS evaluation. In the final experiment, I tested the possibility that this assimilative effect benefits from awareness of the pairings.

\section{Experiment 3}

In Experiment 3, before the categorisation task, instructions manipulated the participants' prior knowledge of the contingency. That was a direct manipulation of contingency awareness. If contingency awareness can contribute to the effect examined in the present research, more accurate prior knowledge would increase the effect of paired valence on the evaluation of the neutral stimulus. Inducing awareness circumvents the interpretation perils of using retrospective contingency memory measure as a measure of contingency awareness (Gawronski \& Walther, 2012).

\section{Method}

Participants. 541 participants (329 Females, $\left.M_{a g e}=36.71, S D_{a g e}=13.5\right)$ started the study, and 378 participants completed all the measures. The analyses did not include five participants with correct-rates below 70\% - 2 SDs below the average correctresponse rate in the categorisation task. The final sample had 373 participants $(228$ Females, $\left.M_{\text {age }}=36.9, S D_{\text {age }}=13.5\right)$. The sample provided $86.5 \%$ power for detecting a moderation effect of $\eta_{p}{ }^{2}=.03$, and $80 \%$ power for detecting an effect of $\eta_{p}{ }^{2}=.025$. 


\section{Design, Materials, and Procedure}

The design was 3 (contingency information: complete, partial, none; between participants) x 2 (paired valence: positive, negative; within participants) x 2 (measures order: evaluation first, memory first). The materials and procedure were identical to those of the constant key-assignment evaluative categorisation condition in Experiment 1, with the following modifications. The instructions were modified by the contingency information condition. In the full-information condition, the following information was added (the specific CS words changed by random assignment):

For your information: the nonsensical words PEEMING and DRIBS always appear before pleasant images, and the words HUBE and THEETS always appear before unpleasant images. You can use that information to respond faster.

In the partial-information condition, the information was:

For your information: two of the words always appear before pleasant images, and two of the words always appear before unpleasant images. You can use that information to respond faster.

In the no-information condition, the instructions were identical to those provided in Experiment 1, not mentioning any contingencies. The only modification in the categorisation task was that participants had a deadline of $2000 \mathrm{~ms}$ to categorise the target stimulus (rather than 3300). Finally, a memory and an evaluation measures appeared in a random order. 
RUNNING HEAD: CONTINGENCY IN EVALUATIVE CATEGORISATION

Evaluation. For each CS (presented in a random order) participants responded to the question Based only on visual appearance, how pleasant is this word's appearance? with the same 1-9 scale used for that question in Experiment 1.

Memory. For each CS (presented in a random order) participants responded to the question During the sorting task, did this word appear before pleasant or unpleasant images? The response scale had nine responses (coded 1-9): Before unpleasant images (certain), Before unpleasant images (pretty sure), Before unpleasant images (probably), Before unpleasant images (guessing), Before an equal number of pleasant and unpleasant images, Before pleasant images (guessing), Before pleasant images (probably), Before pleasant images (pretty sure), and Before pleasant images (certain).

\section{Results}

Figure 3 presents the distribution of the average rating of the CS and the average response to the memory question about the CS by paired-valence, contingency information, and measures order. For statistical inference about the effects of the manipulations on memory and evaluation, each score was subjected to a repeatedmeasures ANOVA with those three factors.

Memory. More positive memory scores reflected more confident memory that the CS preceded positive stimuli. The ANOVA found a main effect for paired-valence, $F(1$, $367)=140.45, p<.001, \eta_{p}{ }^{2}=.28$, reflecting more confident memory that $\mathrm{CS}_{\text {pos }}$ preceded positive stimuli $(M=5.50, S D=1.81)$, than that $\mathrm{CS}_{\text {neg }}$ preceded positive stimuli $(M=$ 3.89, $S D=1.60$ ). A significant paired-valence by contingency-information interaction, 
$F(2,367)=10.51, p<.001, \eta_{p}^{2}=.05$, suggested that the paired-valence effect was stronger (i.e., memory was better) when participants received more accurate contingency information. When the no-information condition was omitted from the ANOVA, the interaction effect, $F(1,254)=7.60, p=.006, \eta_{p}{ }^{2}=.03$, indicated a stronger pairedvalence effect in the full-information $\left[F(1,117)=68.58, p<.001, \eta_{p}{ }^{2}=.37\right]$, than in the partial-information condition $\left[F(1,137)=55.20, p<.001, \eta_{p}{ }^{2}=.29\right]$. When the fullinformation condition was omitted from the ANOVA, an interaction effect, $F(1,250)=$ $4.17, p=.042, \eta_{p}{ }^{2}=.02$, indicated a stronger paired-valence effect in partial-information condition than in the no-information condition $\left[F(1,113)=20.69, p<.001, \eta_{p}{ }^{2}=.15\right]$. These results suggest that the induction of contingency awareness was successful.

A third significant effect in the main ANOVA was the interaction between paired-valence and measures-order, $F(1,367)=7.56, p=.006, \eta_{p}{ }^{2}=.02$, reflecting stronger paired-valence effect (i.e., better memory) when the memory measure appeared right after the categorisation task, $F(1,156)=82.03, p<.001, \eta_{p}{ }^{2}=.34$, than when the evaluation measure appeared before the memory measure, $F(1,211)=53.89, p<.001$, $\eta_{p}^{2}=.20$

Evaluation. The repeated-measures ANOVA found only two significant effects: a main effect for paired-valence, $F(1,367)=44.20, p<.001, \eta_{p}{ }^{2}=.11$, reflected the expected effect of pairing - more positive evaluation of $\mathrm{CS}_{\mathrm{pos}}(M=4.90 ; S D=1.80)$ than $\mathrm{CS}_{\text {neg }}(M=4.12 ; S D=1.61)$. A main effect of measures order, $F(1,367)=5.74, p=.017$, $\eta_{p}{ }^{2}=.02$, reflected more liking of all the CSs when participants reported their memory before evaluating the CSs. This unexpected effect seems unrelated to the research 
questions. All the other effects and interactions had $\eta_{\mathrm{p}}{ }^{2}<.01$ and $p>.229$. Most importantly, contingency information did not moderate the effect of paired-valence, $F(2$, $367)=0.35, p=.706, \eta_{p}{ }^{2}<.01, B F_{01}=26.69($ suggesting strong evidence against moderation). Numerically, the effects of paired valence was the strongest in the partialinformation condition, $F(1,137)=21.59, p<.001, \eta_{p}{ }^{2}=.14$, and nearly identical in the full-information condition, $F(1,117)=13.24, p=.001, \eta_{p}{ }^{2}=.10$, and the no-information condition, $F(1,113)=11.08, p=.001, \eta_{p}{ }^{2}=.09$, despite clear difference in contingency memory between these conditions.

For comparison of each two contingency information conditions, I repeated the same ANOVA, each time omitting one contingency information condition. Contingency information did not moderate the effect of paired-valence when the full-information condition was omitted, $F(1,250)=0.73, p=.393, \eta_{p}^{2}<.01, B F_{01}=5.54$, when the partial-information condition was omitted, $F(1,230)=0.17, p=.677, \eta_{p}^{2}<.01, B F_{01}=$ 7.03, and when the no-information condition was omitted, $F(1,254)=0.16, p=.685, \eta_{p}^{2}$ $<.01, B F_{01}=7.04$. In all these analyses, the Bayes factors indicated moderate evidence against moderation by contingency information. In summary, the evidence suggests that adding knowledge about the CS-valence contingency did not increase the assimilative effect of the contingency on the evaluation of the CS. 


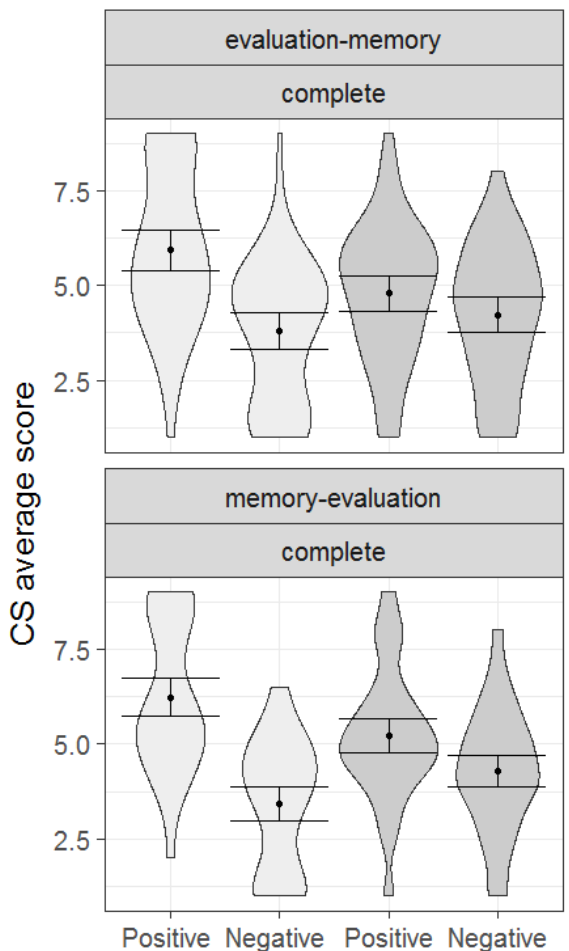

Positive Negative Positive Negative
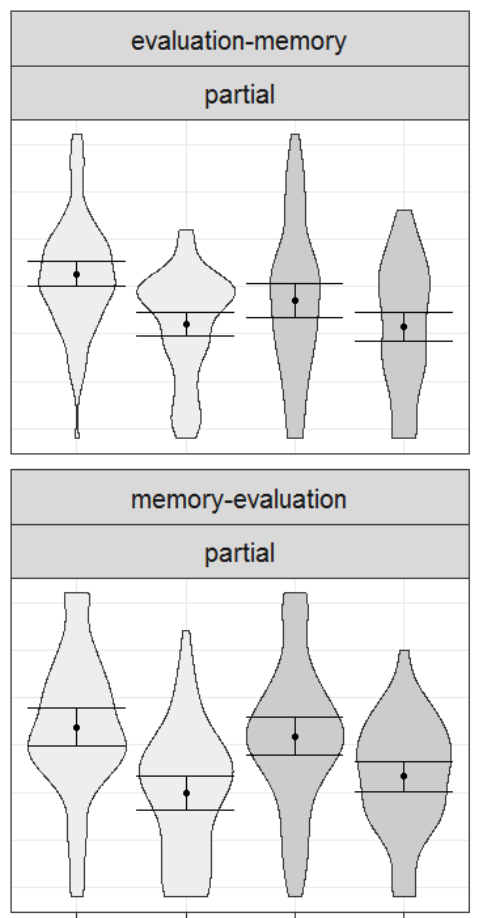

Positive Negative Positive Negative US valence
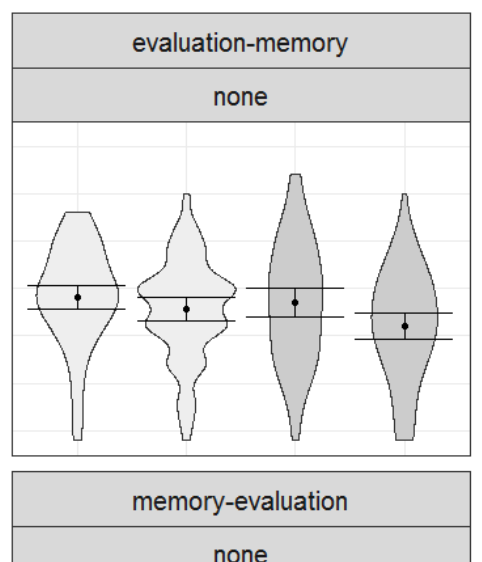

easure Order

- Memory

- EValuation

Figure 3. Evaluative ratings and memory scores for the CS by measure-order, provided information, and valence; Black dot $=$ mean, Error bars $=95 \%$ confidence interval, violins $=$ vertical density plots .

\section{General Discussion}

In three experiments, the pairing of neutral stimuli with affective stimuli that were evaluatively categorised as pleasant or unpleasant changed the evaluation of the neutral stimuli - the evaluation matched the valence with which each neutral stimulus was paired. These results are compatible with previous findings (Schmidt \& De Houwer, 2012). Each experiment added knowledge about the factors that contribute to that effect.

In Experiment 1, the effect decreased when participants categorised the USs by gender, 
and when the mapping of the evaluative response to a motor response was not constant throughout the task. This decrease suggests that pairing with a motor response that comes to signify an evaluative categorisation of a certain valence contributed to the assimilative effect. This evidence is compatible with findings that pairing with an evaluative response causes an assimilative effect (Gast \& Rothermund, 2011a), but it emphasizes the role of coupling the evaluative response with a motor response. The results suggest that when people categorise affective stimuli, and neutral stimuli co-occur with the affective stimuli, EC (the effect of stimulus co-occurrence) is not the only factor that contributes to the assimilative effect on the evaluation of the neutral stimuli.

One alternative account for the increase in the assimilative effect when the evaluative response was constantly mapped to motor response is that the advantage of pairing with a motor response is not the pairing itself but the reduced difficulty in the categorisation task. Indeed, response latency was faster and accuracy was higher when each categorisation response was consistently mapped to one motor response. However, the change in CS evaluation was not stronger when gender categorisation mapped consistently to the same motor response than when gender categorisation was not mapped to a constant motor response. Therefore, reduced cognitive load seems a less likely reason than pairing with a motor response that came to signify certain valence.

In Experiment 1, when participants categorised the stimuli evaluatively but the categorisation was not mapped to a constant motor response, the assimilative effect (in this case, EC) was not stronger than EC in the context of gender categorisation. This result might suggest that focus on valence is not one of the factors that contributes to the 
assimilative effect investigated in the present research. That evidence is incompatible with previous findings that focus on valence during exposure to stimulus co-occurrence increases EC (Gast \& Rothermund, 2011b). Further, whereas previous research found EC only when the main task focused participants on a non-evaluative dimension, the present research found EC even when participants categorised the USs by gender. This evidence might suggest that focus on valence is not a necessary condition for EC. An alternative possibility is that gender is not an entirely non-evaluative dimension. Further research with other non-evaluative categorisation dimensions is needed for providing better knowledge whether co-occurrence with valence affects evaluation only when people focus on valence.

After finding that pairing with a motor response mapped to one valence contributes to the present effect, Experiment 2 examined whether that factor replaces the effect of stimuli co-occurrence or adds to it. When a stimulus predicts a US and an evaluative categorisation response mapped to a constant motor response, does cooccurrence with the US still influence evaluation? Experiment 2 found that the assimilative effect was stronger when the US was more extreme. This result suggests that the co-occurrence with the US (i.e., EC) still contributes to the assimilative effect, even when the CS co-occurs with an evaluatively-charged motor response. This finding is important because there is very little evidence that EC occurs when the learning experience includes other factors that influence evaluation (Moran, Bar-Anan, \& Nosek, 2016). Most EC studies have refrained from confounding EC with other possible evaluation effects. As EC research matures, however, it is important to examine its role in complex contexts that provide other evaluatively-charged experiences and information. 
The results of Experiment 2 also suggest that the categorisation context does not cast people into a dichotomous state of mind that sees only positive versus negative valence (Scherer \& Lambert, 2009), with no sensitivity to differences within the same valence category. EC shows sensitivity to US extremity and not only to the US valence category.

Experiment 2's results do not speak to the question whether EC is the result of a Stimulus-Stimulus or a Stimulus-Response link. The effect of stimulus extremity could reflect each of those links. Experiment 2's results mean that whatever causes EC when there is no US categorisation, still influences the CS evaluation, even when the CS-US co-occurrence happens in the context of an evaluative categorisation task. In other words, whereas Experiment 1's results suggest that EC (change in CS evaluation after CS-US cooccurrence) is not the only cause for the change in CS evaluation when the USs are evaluatively categorised, Experiment 2's results suggest that EC is still one of the causes for the CS evaluation change in that context.

Finally, Experiment 3 found that contingency awareness is not one of the factors that contribute to the assimilative effect of pairing with valence in an evaluative categorisation task. Providing participants, in advance, complete or partial information about the co-occurrence with valence improved retrospective memory of the cooccurrence, lending strong evidence that the information increased contingency awareness during the pairing task. Nevertheless, there was no evidence that an increase in contingency awareness increased the evaluative effect. This suggests that contingency awareness does not contribute to the effect of pairing with valence on evaluation. 
However, the statistical inference was only of moderate strength. This was a null finding with a sample size that provided $80 \%$ power for detecting moderation by contingency knowledge of $\eta_{p}{ }^{2}=.025$. A Bayesian analysis favoured the null hypothesis with moderate strength. Further support for the validity of Experiment 3's results comes from a previous study that paired CSs and USs that were also evaluatively categorised (Schmidt \& De Houwer, 2012). In that study, each CS co-occurred $80 \%$ of its occurrences with US of one valence and 20\% with the other valence. Most participants in that study did not show accurate retrospective co-occurrence memory, and there was no correlation between memory and the change in the CS evaluation. Using a different method that circumvents the inferential problems from retrospective co-occurrence memory measure, the present research strengthens the evidence that in an evaluative categorisation task, awareness might not play a causal role in the change of the CS evaluation. Importantly, because Experiment 2 in the present research found that EC contributes to the change in CS evaluation, Experiment 3's results might suggest that, at least sometimes, awareness does not play a role in EC. These are intriguing results that call for accumulating more evidence with the methodology presented in Experiment 3.

If indeed contingency awareness does not increase the effect of pairing on evaluation, it might suggest that previous findings of positive correlation between contingency memory and evaluation did not reflect a causal relation with awareness as the cause and evaluation as the effect (for evidence of the opposite causal relation, see Bar-Anan \& Amzaleg-David, 2014, and Bar-Anan, De Houwer, \& Nosek, 2010). The present results are incompatible with the propositional perspective on learning (Mitchell, De Houwer, \& Lovibond, 2009). According to that account, explicit knowledge about co- 
occurrence mediates effects of pairing on behaviour. If that was the case, however, an increase in that knowledge should have increased the effect of pairing on evaluation.

\section{Summary}

The present research examined how pairing with affective stimuli influences the evaluation of a neutral stimulus when the perceiver is engaged in categorising the affective stimuli. The results suggest that the evaluation of the neutral stimulus is assimilated to the valence of the co-occurring affective stimuli even when the categorisation is by gender, and even when the motor response that indicates the categorisation changes randomly throughout the task. However, the effect is stronger when the categorisation is evaluative and the motor response is constantly paired with one valence. Pairing with US of more extreme valence also increases the effect. These results suggest that at least two factors contribute to the effect: stimulus co-occurrence and pairing with a motor response that signifies valence. In contrast, the research found no evidence that contingency awareness contributes to the effect.

The categorisation task studied in the present research is a useful tool for investigating evaluative learning: it provides a context in which participants actively engage in actions directed at the affective stimuli (rather than passively observe the stimuli). In the context of an active task, it is easy to manipulate various important factors such as goals, attention, difficulty, importance, and motivation. It is also easy to add factors other than stimulus co-occurrence (e.g., pairing with a motor response). The present study investigated the psychological processes that change evaluation in this task, 
advancing the understanding of evaluative learning in complex conditions. Further research with such tasks could further increase that understanding.

\section{References}

Bar-Anan, Y., \& Amzaleg-David, E. (2014). The effect of evaluation on co-occurrence memory judgment. Cognition and Emotion, 28, 1030-1046.

Bar-Anan, Y., De Houwer, J., \& Nosek, B. A. (2010). Evaluative conditioning and conscious knowledge of contingencies: A correlational investigation with large samples. Quarterly Journal of Experimental Psychology, 63, 2313-2335.

De Houwer, J. (2006). Using the Implicit Association Test does not rule out an impact of conscious propositional knowledge on evaluative conditioning. Learning and Motivation, 37, 176-187.

De Houwer, J. (2009). The propositional approach to learning as an alternative for association formation models. Learning and Behavior, 37, 1-20.

De Houwer, J., Thomas, S., \& Baeyens, F. (2001). Association learning of Likes and dislikes: A review of 25 years of research on human evaluative conditioning. Psychological Bulletin, 127, 853-869.

Jeffreys, H. (1961). Theory of probability (3rd ed.). Oxford, UK: Oxford University Press

Gast, A., \& Rothermund, K. (2011a). What you see is what will change: Evaluative conditioning effects depend on a focus on valence. Cognition and Emotion, 25, 89-110. 
RUNNING HEAD: CONTINGENCY IN EVALUATIVE CATEGORISATION

Gast, A., \& Rothermund, K. (2011b). Journal of Experimental Psychology: Animal Behavior Processes, .

Gawronski, B., \& Walther, E. (2012). What do memory data tell us about the role of contingency awareness in evaluative conditioning. Journal of Experimental Social Psychology, 48, 617-623.

Kattner, F. (2012). Revisiting the relation between contingency awareness and attention: Evaluative conditioning relies on a contingency focus. Cognition and Emotion, $26,166-175$.

Laham, S. M., Kashima, Y., Dix, J., Wheeler, M., \& Levis, B. (2014). Elaborated contextual framing is necessary for action-based attitude acquisition. Cognition and Emotion, 28, 1119-1126.

Lang, P. J., Bradley, M. M., \& Cuthbert, B. N. (1999). The International Affective Picture System (IAPS): Technical manual and affective ratings. Gainesville, FL: University of Florida, The Center for Research in Psychophysiology.

Mitchell, C. J., De Houwer, J., \& Lovibond, P. F. (2009). The propositional nature of human associative learning. Behavioral and Brain Sciences, 32, 183-198.

Moran, T., Bar-Anan, Y., \& Nosek, B. A. (2016). The assimilative effect of cooccurrence on evaluation above and beyond the effect of relational qualifiers. Social Cognition, 34, 343-356.

Nosek, B. A. (2005). Moderators of the relationship between implicit and explicit evaluation. Journal of Experimental Psychology: General, 134, 565-584.

Olson, M. A., \& Fazio, R. H. (2001). Implicit attitude formation through classical conditioning. Psychological Science, 12, 413-417. 
JASP Team (2016). JASP (Version 0.8.0.0) [Computer software].

Scherer, L. D., \& Lambert, A. J. (2009). Contrast effects in priming paradigms:

Implications for theory and research on implicit attitudes. Journal of personality and Social Psychology, 97, 383-403.

Schmidt, J. R., \& De Houwer, J. (2012). Contingency learning with evaluative stimuli. Experimental Psychology, 59, 175-182.

Sweldens, S., Corneille, O., \& Yzerbyt, V. (2014). The role of awareness in attitude formation through evaluative conditioning. Personality and Social Psychology Review, 18, 187-209.

Rouder, J. N., Morey, R. D., Speckman, P. L., \& Province, J. M. (2012). Default Bayes factors for ANOVA designs. Journal of Mathematical Psychology, 56, 356-374.

Walther, E., Weil, R., \& Langer, T. (2011). Why do we like the iPhone? The role of evaluative conditioning in attitude formation. Social and Personality Psychology Compass, 5, 473-486.

Zlotnick, E., Dzikiewicz, A. J., \& Bar-Anan, Y. (2015). Minno.js (Version 0.3) [Computer software]. 